\title{
Der Erfolg der Sprachtherapie wird verbessert
}

Fragestellung: Führt wiederholte anodale transkranielle Gleichstromstimulation (tDCS) bei Aphasiepatienten zu einem besseren Resultat der Sprachtherapie?

Hintergrund: Eine Reihe von experimentellen und klinischen Studien hat auf einen möglichen therapeutischen Effekt der tDCS im Rahmen der Rehabilitation von Patienten mit einer Aphasie nach Schlaganfall hingewiesen. Aber es fehlen bisher randomisierte und kontrollierte Studien, die einen nachhaltigen Effekt der tDCS belegen können.

Meinzer M, Darkow R, Lindenberg $R$ et al. Electrical stimulation of the motor cortex enhances treatment outcome in poststroke aphasia. Brain 2016: 139; $1152-63$
Patienten und Methodik: In einer randomisierten, doppelt verblindeten und shamkontrollierten Studie erhielten 26 Patienten zweimal täglich über acht Tage eine intensive Sprachtherapie. $\mathrm{Zu}$ Beginn der Therapie erhiel- ten sie jeweils entweder eine 20-minütige anodale tDCS über dem Motokortex oder eine Shamstimulation, bei der die Gleichstromstimulation nach 30 Sekunden wieder langsam ausgeschlichen wurde, um eine Verblindung zu gewährleisten.

Ergebnisse: Die Auswertung der Ergebnisse zeigte, dass diejenigen Patienten, die eine anodale tDCS erhielten, bessere Effekte der Sprachtherapie erzielten. Dieser Vorteil in der verbesserten Benennung von trainierten und untrainierten Objekten der tDCS-Gruppe ließ sich noch sechs Monate nach der Therapie nachweisen. Die tDCS-Therapie führte dabei insgesamt nicht nur zu einer spezifischen Verbesserung in der Benennung, sondern auch zu einer Rehabilitation der alltagsrelevanten kommunikativen Fähigkeiten der Patienten.

Schlussfolgerungen: Die Autoren folgern aufgrund ihrer Ergebnisse, dass mit der nun vereinfachten Motorkortex-Montage eine effektive und leicht zu handhabende Therapie der Aphasie nach Schlaganfall zur Verfügung steht.

\section{- Kommentar von David Liebetanz, Göttingen}

\section{Eine „Hintertür" für die Behandlung von Aphasiepatienten?}

Schwacher Gleichstrom induziert neuroplastische Veränderungen der Erregbarkeit des Kortex. Diese Veränderungen sind abhängig von der Polarität und der Dauer der Gleichstromstimulation sowie von der verwendeten Stromstärke. Die ursprünglich auf tierexperimentelle Untersuchungen aus den 1950er- und 1960er-Jahren zurückgehende Methode konnte zur Jahrtausendwende erfolgreich auf die Anwendung am humanen Kortex übertragen werden. Hierbei führt kathodale transkranielle Gleichstromstimulation (tDCS) zu einer Verminderung und anodale Stimulation zu einer Erhöhung der kortikalen Erregbarkeit.

Hinsichtlich des therapeutischen Potenzials der tDCS liegt eine Vielzahl positiver Pilotstudien zu einer großen Bandbreite an psychiatrischen und neurologischen Erkrankungen vor. Insbesondere die durch den Schlaganfall pathologisch veränderte kortikale Erregbarkeit auf ipsi- und kontralateraler Seite war das Ziel vielfältiger präklinischer und klinischer Untersuchungen. Bei allem Optimismus fehlte jedoch bisher der Nachweis einer klinischen Wirksamkeit auf dem Niveau eines RCT. Solche tDCS-Studien sind aber alles andere als trivial in der Durchführung, da es sich um eine mehr oder weniger fokale physikalische Therapie handelt, die auf die individuelle Größe und Lokalisation der Schlaganfallläsion hin justiert werden sollte. Dieses Problem umging die Berliner Arbeitsgruppe allerdings elegant mit einem prinzipiellen Strategiewechsel, der die Bedingungen und Handhabung der therapeutischen tDCS beim Schlaganfall und somit das Studiendesign entscheidend vereinfachen sollte: Nicht das betroffene und durch den Schlaganfall "mindererregte" Sprachareal, sondern das benachbarte primärmotorische Handareal wurde mit Gleich- strom stimuliert. Dieses war bei keinem Patienten betroffen. Es wurde argumentiert, dass die Stimulation des Motokortex bei den Patienten eine Modulation von funktionell verbundenen prämotorischen und frontalen Hirnregionen ermöglicht, die in der Sprachproduktion involviert sind. Durch das Ausweichen auf den nicht betroffenen Motokortex kann auf eine aufwändige, durch Bildgebung unterstützte Individualisierung des Therapieregimes verzichtet werden. Daher folgern die Autoren, dass hier eine vielversprechende "Hintertür-"Therapie für Aphasiepatienten zur Verfügung steht.

Ob die erzielten Verbesserungen der Therapieergebnisse tatsächlich auf eine Modulation der unterstützenden Rolle des primärmotorischen Kortex zurückzuführen oder ob die Effekte einer relativen Unfokalität der tDCS zuzuschreiben sind, bleibt noch zu untersuchen. Auch werden weitere Studien zeigen müssen, ob sich die therapieassoziierte Verbesserung der Objektbenennung auch auf andere Domänen der Sprache übertragen lässt.

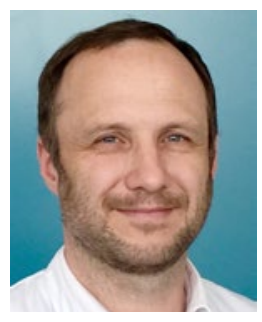

Prof. Dr. med. David Liebetanz, Göttingen

Universitätsmedizin Göttingen Klinik für Klinische Neurophysiologie E-Mail: clneuphy@gwdg.de 